\title{
Effects of forkhead box protein M1 on trophoblast invasion and its role in preeclampsia development
}

\author{
XUENA CUI, JIN'E XU, YUZHI JI, XIUHONG SONG, JUNHUAN WANG, \\ LIJUAN ZHANG, SHENGMEI YANG and YUANHUA YE
}

Department of Obstetrics, The Affiliated Hospital of Qingdao University, Qingdao, Shandong 266000, P.R. China

Received March 9, 2017; Accepted January 3, 2018

DOI: $10.3892 /$ etm.2018.6195

\begin{abstract}
The present study aimed to investigate the expression of the forkhead box protein M1 (FOXM1) in the placenta of patients with preeclampsia, and its effect on trophoblasts. A total of 28 patients with preeclampsia and 30 patients without preeclampsia (controls) who underwent cesarean section and were admitted to the Affiliated Hospital of Qingdao University between June 2013 and September 2016 were enrolled in the present study. The expression of FOXM1 in placental tissues was examined by reverse transcription-quantitative polymerase chain reaction, western blotting and immunohistochemistry. HTR8/SVneo cells were used to measure the in vitro expression of the vascular endothelial growth factor (VEGF). The results demonstrated that FOXM1 expression was downregulated in the placental tissues of patient with preeclampsia $(\mathrm{P}<0.05)$. Following the silencing of FOXM1 expression, the proliferation of HTR8/SVneo cells was suppressed. The results of flow cytometry demonstrated that proportion of HTR8/SVneo cells in the G0/G1 phase and the proportion of apoptotic cells increased. The expression of the apoptosis regulator BCL-2, as well as the expression of VEGF mRNA and protein expression were also downregulated following FOXM1 silencing. FOXM1 may therefore promote the development of preeclampsia via the VEGF signaling pathway.
\end{abstract}

\section{Introduction}

Preeclampsia is one of the main causes of perinatal maternal and infant mortality, with an incidence of $1-5 \%(1,2)$. Pathophysiological changes begin in early pregnancy; however symptoms usually become evident after 20 weeks gestation (3). The main symptoms of preeclampsia include hypertension, proteinuria, edema, full body spasms and in severe cases,

Correspondence to: Professor Yuanhua Ye, Department of Obstetrics, The Affiliated Hospital of Qingdao University, 16 Jiangsu Road, Qingdao, Shandong 266000, P.R. China E-mail: 18661805507@163.com

Key words: forkhead box protein M1, trophoblast, vascular endothelial growth factor, preeclampsia multiple-organ dysfunction (3). Preeclampsia is a condition associated with the placenta; clinical practice has demonstrated that disease-associated symptoms are rapidly relieved following placental delivery (4). Decreased trophoblast invasion, required to establish pregnancy, contributes to the development of preeclampsia (5) and inhibits vascular reconstruction in the uterine spiral arteries (6). Preeclampsia is a complex disease and its course is difficult to predict, therefore the termination of pregnancy may be recommended as a method of controlling disease progression (7). The etiology and pathogenesis of preeclampsia remains unclear.

Forkhead box protein M1 (FOXM1) is a protein expressed in a number of mammalian cells and serves important roles in physiological functions and pathological conditions, including mitosis, cell proliferation, differentiation, senescence, organogenesis, DNA repair and cancer invasion (8-10). FOXM1 in villous and decidual tissue is expressed in the cytotrophoblast during early pregnancy, and may affect embryo implantation and early placental formation (11-13). It has been demonstrated that the vascular endothelial growth factor (VEGF) signaling pathway is involved in the function of extravillous trophoblasts and trophoblast invasion $(14,15)$. Furthermore, VEGF promotes endothelial cell division, vascular endothelial cell proliferation and migration, and tubular structure formation during neovascularization. It also enhances the permeability of vascular endothelial cells (16). Therefore, the current study investigated whether FOXM1 involvement during preeclampsia occurs via the VEGF signaling pathway.

In the present study, the mRNA and protein expression of FOXM1 in the placenta of patients with or without preeclampsia were measured by reverse transcription-quantitative polymerase chain reaction (RT-qPCR) and western blotting. In vitro function experiments were performed in HTR8/SVneo trophoblast cells. The association between FOXM1 and VEGF was investigated to explore the role of FOXM1 in preeclampsia.

\section{Patients and methods}

Patients. A total of 28 pregnant patients with preeclampsia and 30 patients without preeclampsia who underwent cesarean sections at the Affiliated Hospital of Qingdao University between June 2013 and September 2016 were enrolled in the present study. The patients without preeclampsia were used 
as controls. Participants were all female, with a median age of 33 years (range, 21-41 years) in the preeclampsia group and 35 years (range, 20-42 years) in the control group. The diagnosis of preeclampsia was made by professors from the Department of Pathology of the Affiliated Hospital of Qingdao University (Qingdao, China). This diagnosis was confirmed by serological tests and other clinical examinations, including blood pressure assessment, routine laboratory evaluation (the measurement of urine volume, creatinine, alanine aminotransferase or aspartate aminotransferase, and platelets), fetal status assessment and proteinuria detection. The mean weights of infants from the control and preeclampsia groups were $3.15 \pm 0.97$ and $2.79 \pm 1.03 \mathrm{~kg}$, respectively. The blood pressure of infants was not measured. Placental tissue samples (500 mg) were collected from each participant during cesarean section and stored in liquid nitrogen at $-196^{\circ} \mathrm{C}$. The protocol of the present study was approved by the Ethical Review Board of the Affiliated Hospital of Qingdao University and written informed consent was obtained from all patients.

Reagents and equipment. SuperReal PreMix (SYBR-Green; cat. no. FP204) and the TIANScript II RT kit (cat. no. KR107) were purchased from Tiangen Biotech Co.,Ltd. (Beijing, China). $\mathrm{iQ}^{\mathrm{TM}}$ and Image Lab ${ }^{\mathrm{TM}}$ software (version 3.0) were purchased from Bio-Rad Laboratories, Inc. (Hercules, CA, USA) for RT-qPCR. The BCA protein assay kit (cat. no. RTP7102) was purchased from Real-Times (Beijing) Biotechnology Co., Ltd. (Beijing, China). Cell preservation solution was purchased from Ruikangdi Co., Ltd. (Beijing, China). Enhanced chemiluminscence (ECL) solution (cat. no. ab65623) was purchased from Abcam (Cambridge, MA, USA). All plasmids were designed and synthesized by Sangon Biotech Co., Ltd. (Shanghai, China). Cell proliferation and cytotoxicity test kits for the MTT assay were purchased from JRDUN Biotechnology Co., Ltd. (Shanghai, China). Cell cycle and apoptosis detection kits were purchased from Beyotime Institute of Biotechnology (Jiangsu, China). The Annexin V-FITC apoptosis detection kit was purchased from Vazyme Biotech, Co., Ltd. (Nanjing, China).

$R T$-qPCR. Placental tissues were pulverized with liquid nitrogen. A total of $1 \mathrm{ml}$ TRIzol (cat. no. 15596026; Invitrogen; Thermo Fisher Scientific, Inc., Waltham, MA, USA) was added to each $100 \mathrm{mg}$ tissue for adequate lysis and RNA was extracted using the phenol chloroform method as previously described (17). The integrity of the RNA bands was detected using agarose gel electrophoresis as previously described (18). RNA purity was assessed by measuring the 260/280 ratio using a spectrophotometer. RT was performed using $1 \mu \mathrm{g}$ total RNA using a TIANScript II RT kit. Template cDNA was stored at $-20^{\circ} \mathrm{C}$. qPCR was performed using the SuperReal PreMix (SYBR-Green) and specific primers. The primers for FOXM1 were as follows: Forward, 5'-GGCTCCCGCAGC ATCAAGCA-3' and reverse, 5'-TGTTCCGGCGGAGCTCT GGA-3'. The primers for $\beta$-actin, which was used as the internal reference gene, were as follows: Forward, 5'-ATCTGGCAC CACACCTTCACAATGAGCTGCG-3' and reverse, 5'-CGT CATACTCCTGCTTGCTGATCCACATCTGC-3'. The primers for VEGF were as follows: Forward, 5'-TTGCCTTGC TGCTCTACCTC-3' and reverse, 5'-AAATGCTTTCTCCGC TCTGA-3'. The qPCR reaction conditions were as follows:
Initial denaturation for $2 \mathrm{~min}$ at $94^{\circ} \mathrm{C}$, followed by 35 cycles of denaturation for $30 \mathrm{sec}$ at $94^{\circ} \mathrm{C}$, annealing for $30 \mathrm{sec}$ at $55^{\circ} \mathrm{C}$ and extension for $1 \mathrm{~min}$ at $71^{\circ} \mathrm{C}$, and final extension for $2 \mathrm{~min}$ at $71^{\circ} \mathrm{C}$. Relative gene expression of FOXM1 and VEGF was calculated using the $2^{-\Delta \Delta \mathrm{Ca}}$ method (19). All experiments were performed in triplicate.

Western blotting. The expression of FOXM1 and VEGF proteins was determined by western blotting. Total proteins were extracted with radioimmunoprecipitation lysis buffer (cat. no. P0013B; Beyotime Institute of Biotechnology) following the supplier protocol. Protein concentration was determined using a BCA kit. Proteins (50 $\mu \mathrm{g}$ per lane) were separated using $10 \%$ SDS-PAGE and transferred to a polyvinylidene fluoride membrane (EMD Millipore, Billerica, MA, USA). The membrane was blocked with $5 \%$ skimmed milk in Tween-20 in Tris buffer solution at room temperature for $1 \mathrm{~h}$. Proteins were then incubated with rabbit anti-human primary antibodies against FOXM1 (cat. no. ab175798; 1:2,000), VEGF (cat. no. ab46154; 1:1,000), BCL-2 (cat. no. ab32124; 1:1,000) and the internal reference protein $\beta$-actin (cat. no. ab129348; 1:5,000; all Abcam) overnight at $4^{\circ} \mathrm{C}$. Proteins were subsequently incubated with horseradish-conjugated secondary antibodies (goat anti-rabbit; ab6721; 1:3,000; Abcam) at room temperature for $1 \mathrm{~h}$. The membrane was exposed to the ECL solution and then exposed to the Gel Doc XR gel imaging and analysis system (Bio-Rad Laboratories, Inc., Hercules, CA, USA). The image signal was analyzed and quantified by Image Lab software version 3.0 (Bio-Rad Laboratories, Inc.). The relative content of the target protein was calculated by the ratio of the gray value of the target protein band to that of the internal reference band. All experiments were performed in triplicate.

Immunohistochemistry. Placental tissues were fixed in $10 \%$ formaldehyde at $4^{\circ} \mathrm{C}$ for $24 \mathrm{~h}$, embedded in paraffin and cut into $5-\mu \mathrm{m}$-thick sections. Then, sections were dewaxed using xylene and rehydrated in a descending alcohol series. Antigen retrieval was achieved by incubation with sodium citrate solution (Beyotime Institute of Biotechnology) and the sample was heated in the microwave at $97^{\circ} \mathrm{C}$ for $12 \mathrm{~min}$. Following washing of the sections, they were incubated with $3 \%$ hydrogen peroxide at room temperature for $10 \mathrm{~min}$. Following blocking with $10 \%$ goat serum (Beyotime Institute of Biotechnology) at room temperature for $1 \mathrm{~h}$, sections were incubated with rabbit anti-human FOXM1 primary antibodies (cat. no. ab83097; 1:50; Abcam) overnight at $4^{\circ} \mathrm{C}$. Samples were then washed with PBS, incubated with the goat anti-rabbit horseradish peroxidase conjugated secondary antibodies (cat. no. ab6721; 1:1,000; Abcam) for $1 \mathrm{~h}$ at room temperature and washed with PBS. Finally, sections were developed with chromogenic reagent 3'3-diaminobenzidine for $\sim 10 \mathrm{sec}$ at room temperature, counterstained with hematoxylin for $20 \mathrm{sec}$ at room temperature, mounted with neutral gum and observed using a Nikon TS100-F optical microscope (Nikon Corporation, Tokyo, Japan; magnification, x500).

Human HTR8/SVneo transfection. HTR8/SVneo cells were obtained from American Type Culture Collection (Manassas, VA, USA) and cultured at $37^{\circ} \mathrm{C}$ in $5 \% \mathrm{CO}_{2}$ with 
A

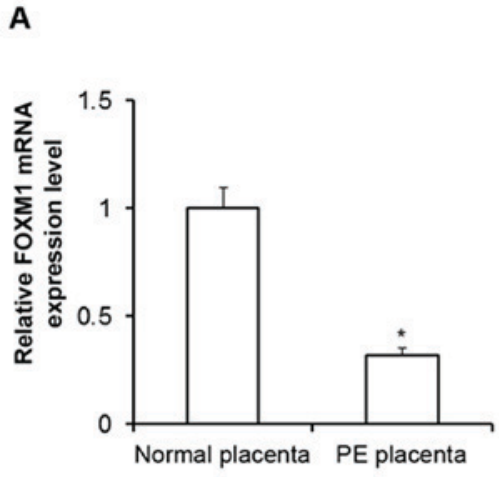

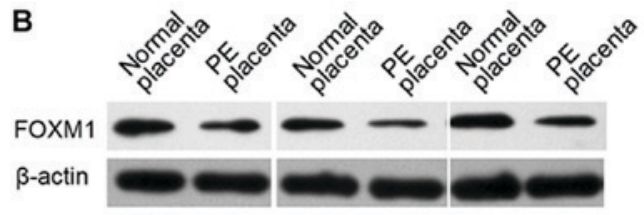

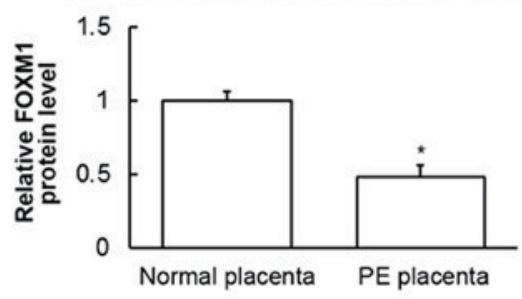

C

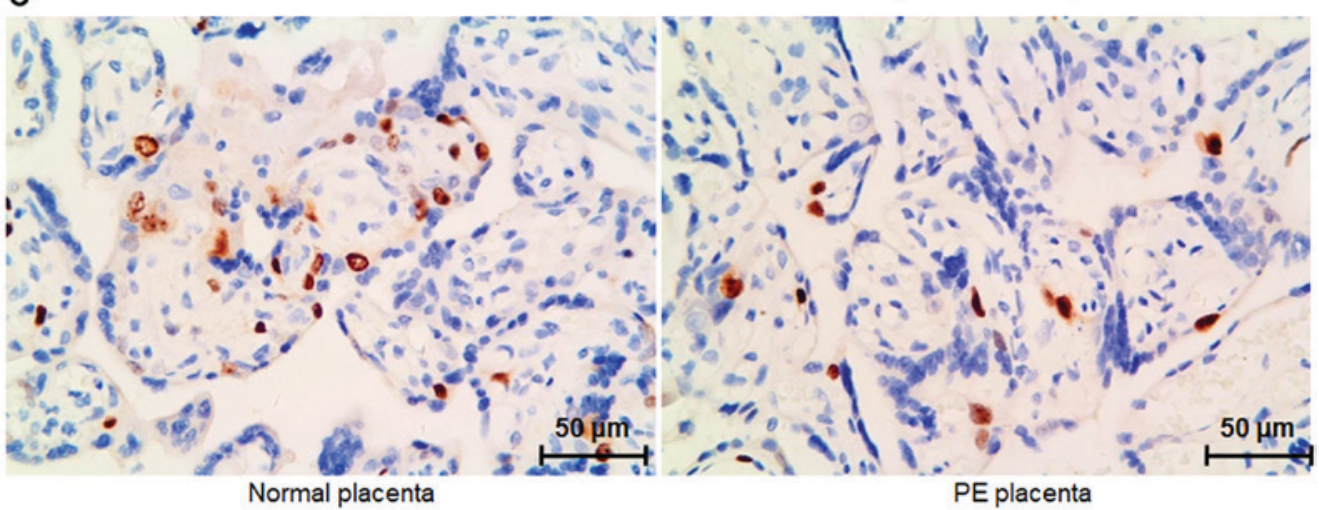

Figure 1. FOXM1 expression is decreased in the placentas of patients with PE. The expression of FOXM1 (A) mRNA and (B) protein was decreased in PE placenta compared with normal placenta. (C) Immunohistochemical staining of FOXM1 expression in placental tissues. "P $<0.05$ vs. normal placenta. PE, preeclampsia; FOXM1, forkhead box protein M1. Tamhane's T2 was used for statistical analysis.

Dulbecco's Modified Eagle's medium (DMEM) and 10\% fetal bovine serum (FBS). Cells were plated in 24-well plates at $3 \times 10^{5}$ cells/well 1 day prior to transfection and cultured in 10\% FBS in F12/DMEM without antibiotics. Transfection was performed when cells reached $70 \%$ confluence. A total of $50 \mathrm{nM}$ FOX1 small interfering (si)RNA (designed by Sangon Biotech Co., Ltd.) was transfected into cells using Lipofectamine ${ }^{\circledR} 2000$ Transfection Reagent (Invitrogen; Thermo Fisher Scientific, Inc.) as previously described (20). The sequence of FOX1 siRNA was forward, 5'-ACCCAAACC AGCUAUGAUGdTdT-3' and reverse, 5'-CAUCAUAGCUGG UUUGGGUdTdT-3'. The sequences of the scramble siRNA were as follows: Forward, 5'-UUCUCCGAACGUGUCACG UdTdT-3' and reverse, 5'-ACGUGACACGUUCGGAGAAd TdT-3'. Levels of FOXM1 mRNA and protein were measured $48 \mathrm{~h}$ following transfection.

HTR8/SVneo cell proliferation following transfection. Cells were plated in triplicate in 96-well plates at a density of $2 \times 10^{3}$ cells/well. Subsequently, $20 \mu 15 \mathrm{~g} / \mathrm{l}$ MTT solution was added at 24, 48 and $72 \mathrm{~h}$. Purple crystals were solubilized by adding $150 \mu \mathrm{l}$ dimethyl sulfoxide and plates were then incubated in a humidified atmosphere at $37^{\circ} \mathrm{C}$ for $4 \mathrm{~h}$. The cell proliferation curve was constructed by measuring the spectrophotometric absorbance of the samples at a wavelength of $490 \mathrm{~nm}$.

Flow cytometric detection of HTR8/SVneo cell cycle and apoptosis post-transfection. At $48 \mathrm{~h}$ post-transfection, cells were collected. Cells $\left(2 \times 10^{5}\right)$ were fixed with $75 \%$ alcohol overnight at $4^{\circ} \mathrm{C}$ and incubated with $1 \mathrm{mg} / \mathrm{ml}$ RNaseA for $30 \mathrm{~min}$ at $37^{\circ} \mathrm{C}$. Cells were stained with propidium iodide (PI) at room temperature for $15 \mathrm{~min}$. Cells were also incubated with Annexin $\mathrm{V}$ at room temperature for $15 \mathrm{~min}$ for apoptosis analysis. The cell cycle and cell apoptosis were analyzed using a flow cytometer.

Statistical analysis. All data were analyzed using SPSS statistical software (version 18.0; SPSS, Inc., Chicago, IL, USA) and were tested for normal distribution. Data are expressed as mean \pm standard deviation. Multiple measurement data were tested by one-way analysis of variance followed by the Least Significant Difference and Student-Newman-Keuls method or Tamhane's $\mathrm{T} 2$, where appropriate. $\mathrm{P}<0.05$ was considered to indicate a statistically significant difference.

\section{Results}

FOXM1 mRNA and protein expression is decreased in the placentas of patients with preeclampsia. The results of RT-qPCR demonstrated that the expression of FOXM1 mRNA in the placental tissue of patients with preeclampsia was significantly decreased compared with the control group $(\mathrm{P}<0.05$; Fig. 1A). The expression of FOXM1 protein in the placental tissue of patients with preeclampsia was also significantly decreased $(\mathrm{P}<0.05$; Fig. 1B). This was supported by the results of immunohistochemical staining, which exhibited decreased staining for FOXM1 in the placental tissues of patients with preeclampsia (Fig. 1C). The results of the current study indicate that the expression of FOXM1 mRNA and protein is 

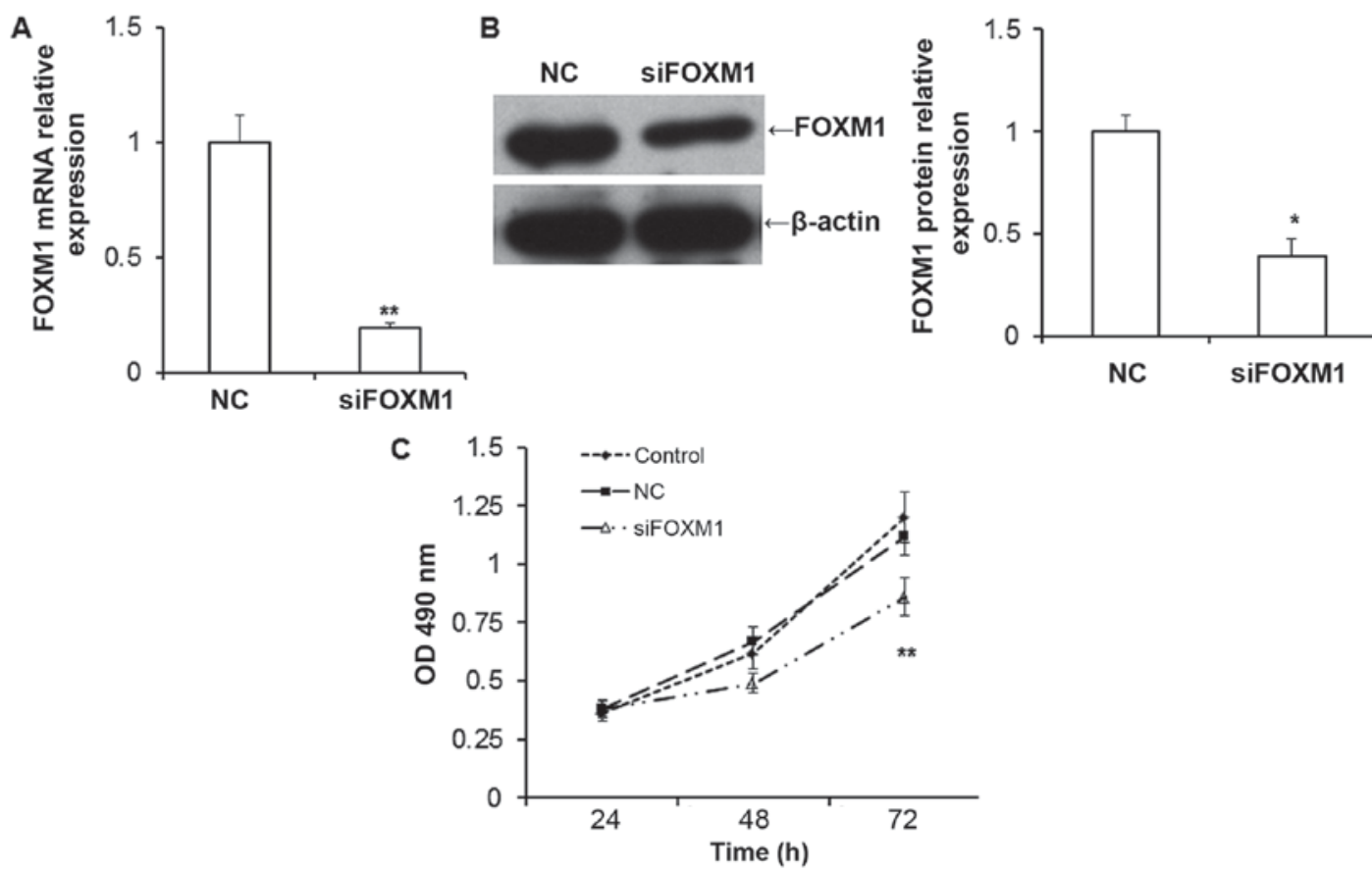

Figure 2. FOXM1 silencing decreases the proliferation of trophoblasts. FOXM1 (A) mRNA and (B) protein expression in HTR8/SVneo cells following transfection with siFOXM1. (C) MTT assay measuring the proliferation of HTR8/SVneo cells following transfection with siFOXM1 or NC. ${ }^{*} \mathrm{P}<0.05$ and ${ }^{* *} \mathrm{P}<0.01$ vs. NC. NC, negative control; FOXM1, forkhead box protein M1; si, small interfering RNA; OD, optical density. Student-Newman-Keuls method was used for statistical analysis.

downregulated in patients with preeclampsia, suggesting that FOXM1 may serve a regulatory role in preeclampsia.

FOXM1 silencing decreases the proliferation of HTR8/SVneo cells. To examine the function of FOXM1 in the placenta, in vitro evaluations were conducted by silencing FOXM1 expression in HTR8/SVneo cells. The expression of FOXM1 mRNA ( $\mathrm{P}<0.01$; Fig. 2A) and protein $(\mathrm{P}<0.05$; Fig. 2B) were significantly downregulated in HTR8/SVneo cells transfected with FOXM1 siRNA compared with negative control cells. The results of the MTT assay demonstrated that the proliferation of HTR8/SVneo cells was inhibited following siFOXM1 transfection compared with cells transfected with the negative control (NC; $\mathrm{P}<0.01$; Fig. 2C). This suggests that the downregulation of FOXM1inhibits the proliferation of HTR8/SVneo cells.

FOXM1 silencing increases the proportion of cells in the G0/G1 phase. Flow cytometry was used to assess the proportion of cells in each stage of the cell cycle (Fig. 3A). The results demonstrated that the proportion of HTR8/SVneo cells in the G0/G1 phase was significantly increased in cells transfected with FOXM1 siRNA $(\mathrm{P}<0.05)$, whereas the proportion of cells in the $\mathrm{G} 2$ and $\mathrm{S}$-phases was decreased (all $\mathrm{P}<0.05$; Fig. 3B). These results indicate that decreased FOXM1 expression may attenuate the cell cycle in HTR8/SVneo cells.

FOXM1 silencing increases the proportion of apoptotic cells and decreases $B C L-2$ protein expression. The proportion of early apoptotic cells and late apoptotic cells in HTR8/SVneo cells was examined using flow cytometry (Fig. 4A). The results revealed that transfection with FOXM1 siRNA significantly increased the proportion of apoptotic cells compared with cells transfected with NC siRNA $(\mathrm{P}<0.01$; Fig. 4B), suggesting that decreased FOXM1 expression may increase apoptosis in HTR8/SVneo cells. The results also revealed that BCL-2 protein expression was significantly decreased following transfection with FOXM1 siRNA ( $\mathrm{P}<0.05$; Fig. 4C), indicating that FOXM1 is associated with BCL-2 expression.

FOXM1 silencing decreases VEGF expression. VEGF is one of the most potent angiogenic factors and promotes angiogenesis, which, in turn increases blood supply (21). The reduction in VEGF expression leads to the premenstrual onset of preeclampsia, therefore the current study examined the association between FOXM1 and VEGF. The expression of VEGF mRNA and protein in HTR8/SVneo cells was determined by RT-qPCR and western blotting, respectively. The results demonstrated that the decrease of FOXM1 expression significantly downregulated the expression of VEGF mRNA and protein (both $\mathrm{P}<0.05$; Fig. $5 \mathrm{~A}$ and $\mathrm{B}$ ), suggesting that FOXM1 and VEGF may function in concert and that changes in the expression of either protein may induce vascular dysfunction. VEGF receptor (VEGFR) expression in placental tissue samples were also examined in a preliminary experiment by our group, however, the results revealed no significant changes in patients with preeclampsia compared with controls (data not shown).

\section{Discussion}

In the present study, the role of placental FOXM1 expression in the pathogenesis of preeclampsia was investigated. The expression of FOXM1 mRNA and protein was examined in the placenta tissues of patients with or without preeclampsia that underwent cesarean sections. The association between 

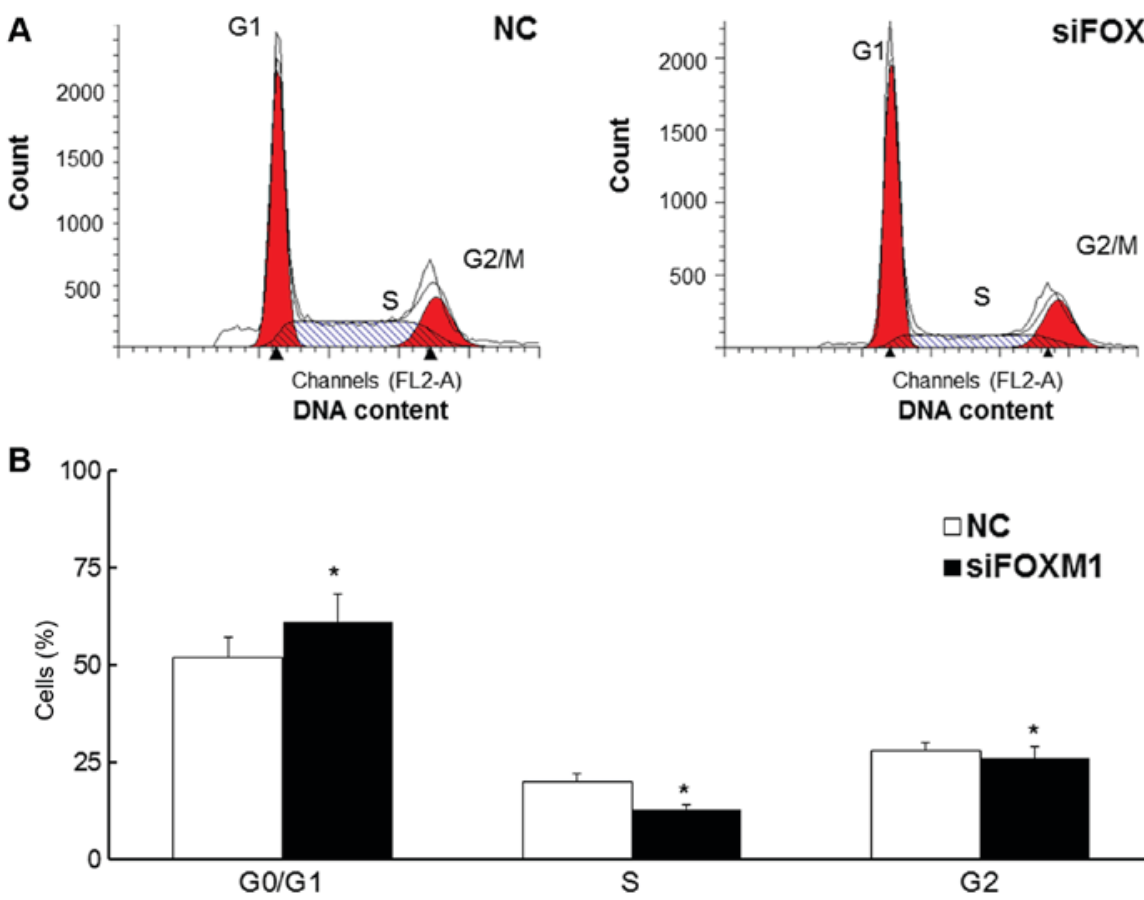

\section{$\square \mathrm{NC}$ - SIFOXM1}

Figure 3. FOXM1 silencing increases the proportion of cells in the G0/G1 phase. (A) Cell cycle distribution and (B) quantification of cell cycle distribution of HTR8/Svneo cells following FOXM1 siRNA transfection. "P<0.05 vs. NC. NC, negative control; FOXM1, forkhead box protein M1; si, small interfering. Student-Newman-Keuls method was used for statistical analysis.
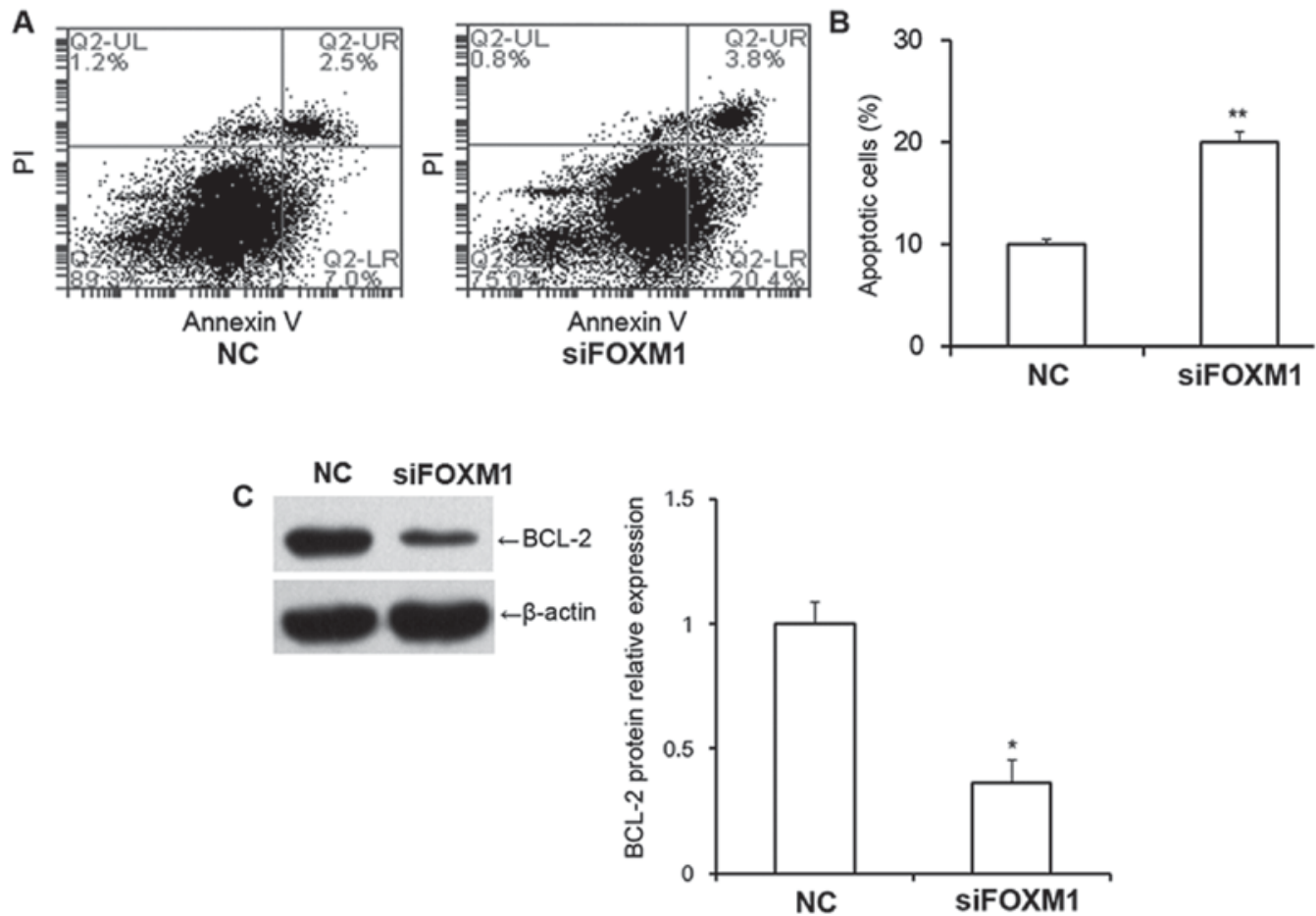

Figure 4. FOXM1 silencing increases the proportion of apoptotic cells and decreases BCL-2 protein expression. (A) Flow cytometric analysis identifying the (B) proportion of apoptotic cells in HTR8/SVneo cells following transfection with FOXM1 siRNA. (C) BCL-2 protein expression in transfected cells. "P<0.05 and ${ }^{* *} \mathrm{P}<0.01$ vs. NC. NC, negative control; FOXM1, forkhead box protein M1; si, small interfering RNA; BCL-2, apoptosis regulator BCL-2; PI, propidium iodide. Student-Newman-Keuls method was used for statistical analysis.

FOXM1 and VEGF was further investigated by performing in vitro HTR8/SVneo trophoblast cell cycle studies.

The primary manifestations of preeclampsia include the shallow invasion of trophoblasts into the endometrium, cessation of vascular remodeling in the uterine spiral arteries and the decreased surface area and density of the villi. Patients with preeclampsia often exhibit disordered vascular remodeling $(22,23)$. Placental hypoperfusion causes placental 
A

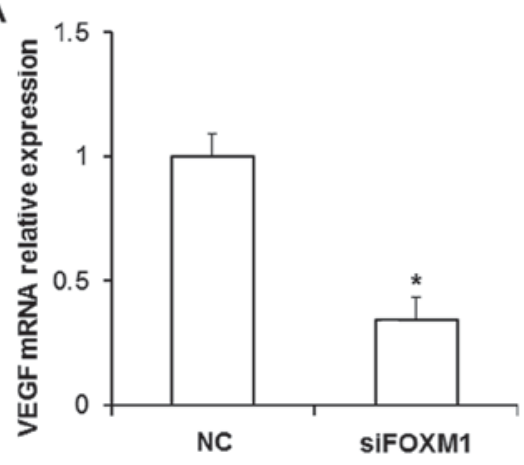

B

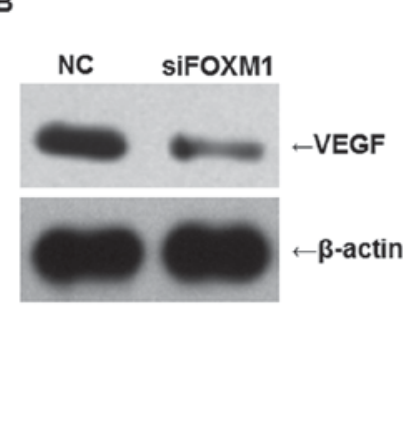

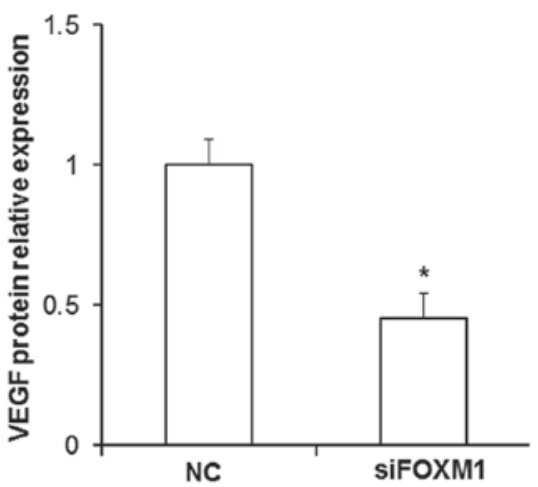

Figure 5. FOXM1 silencing decreases VEGF expression. The expression of VEGF (A) mRNA and (B) protein was decreased in HTR8/SVneo cells following transfection with FOXM1 siRNA. ${ }^{*} \mathrm{P}<0.05$ vs. NC. NC, negative control; FOXM1, forkhead box protein M1; si, small interfering RNA; VEGF, vascular endothelial growth factor. Student-Newman-Keuls method was used for statistical analysis.

hypoxia, metabolism disorders, placental sourced toxicity, vascular endothelial injury and an imbalance of vasoactive substances, which can eventually lead to the development of preeclampsia (24). It is widely accepted that preeclampsia develops in two different stages $(25,26)$. During the first stage, vascular remodeling disorders cause a decrease in villous trophoblast invasion and induce placental hypoxia. In the second stage, multiple peptides produced by the placenta are secreted into the blood circulation of the mother and reactive oxygen species (ROS) build up in the vascular endothelium. The imbalance between ROS production and the antioxidant defense system exacerbates systemic small artery injury, leading to ROS accumulation, hypertension and proteinuria (27). Previous studies have suggested that preeclampsia may be associated with decreased placental trophoblast invasion, ischemia and hypoxia, increased apoptosis, abnormal lipid metabolism and other placental dysfunctions during pregnancy (28-30). It has been reported that the extravillous trophoblasts serve an important role in the process of recoil in spiral arteries; the diminished invasion of extravillous trophoblast cells leads to abnormal vascular remodeling and shallow embryo implantation and ultimately, the development of preeclampsia $(22,31)$.

FOXM1 is highly expressed in a number of malignancies and is involved in tumor cell proliferation, apoptosis, invasion and metastasis; it is therefore a marker of tumor development (32-35). The biological characteristics of trophoblasts in embryos are very similar to those of cancer cells and a number of factors involved in cancer invasion, including human leukocyte antigen and DNA methyltransferase 3A, also serve a role in trophoblast invasion $(36,37)$. The primary difference is that trophoblast invasion is a tightly regulated process (38). FOXM1 is expressed in the cytotrophoblast of villus and decidual tissue during early pregnancy $(11,39)$. FOXM1 is also expressed in the maternal decidual cells and FOXM1 may serve as an indirect regulator of trophoblast invasion via paracrine signaling (40). These results suggest that FOXM1 is involved in the development and progression of preeclampsia.

In the present study, the expression of FOXM1 was measured in normal and preeclampsia placental tissues. The expression of FOXM1 mRNA and protein was significantly decreased in patients with preeclampsia. This suggests that FOXM1 may indirectly regulate trophoblast invasion through paracrine signaling, as decreased FOXM1 expression decreases trophoblast invasion and attenuates the invasion of trophoblast cells into the endometrium $(38,40)$. To the best of our knowledge, the present study is the first to report the abnormal expression of FOXM1 in preeclampsia placenta.

To further investigate the effect of FOXM1 on trophoblast cells, FOXM1 siRNA was transfected into human trophoblast cells, HTR8/Svneo. The results of the MTT assay demonstrated that FOXM1 silencing attenuates the proliferation of HTR8/Svneo cells. Additionally, the results of flow cytometry demonstrated that the decreased expression of FOXM1 blocks the cell cycle at the G0/G1 phase and increases the apoptosis of HTR8/Svneo cells. Furthermore, the results of RT-qPCR and western blotting revealed that decreased FOXM1 expression leads to the downregulation of VEGF mRNA, and VEGF and BCL-2 proteins. VEGF is one of the most effective angiogenic growth factors; it promotes angiogenesis and increases blood supply (41). It has been demonstrated that suppression of the VEGF signaling pathway is associated with the development of preeclampsia (42), which may partially explain the development of vascular remodeling disorder in patients with preeclampsia. Silencing FOXM1 decreases the expression of VEGF, suggesting that FOXM1 may serve its role via the VEGF signaling pathway. VEGFR expression in tissue samples was also examined in a preliminary experiment by our group, however the results revealed no significant changes in patients with preeclampsia compared with healthy patients, which may be a result of other biological functions.

In conclusion, the results of the present study demonstrate that the altered expression of FOXM1 may cause changes in the expression of associated proteins, resulting in decreased trophoblast cell proliferation, prolonged cell cycle and increased cellular apoptosis. This demonstrates that FOXM1 serves an important role in the development of preeclampsia by regulating the VEGF signaling pathway.

\section{References}

1. Cetin O, Guzel Ozdemir P, Kurdoglu Z and Sahin HG: Investigation of maternal psychopathological symptoms, dream anxiety and insomnia in preeclampsia. J Matern Fetal Neonatal Med 30: 2510-2515, 2017. 
2. Baumwell S and Karumanchi SA: Preeclampsia: Clinical manifestations and molecular mechanisms. Nephron Clin Pract 106: c72-c81, 2007.

3. Sones JL and Davisson RL: Preeclampsia, of mice and women. Physiol Genomics 48: 565-572, 2016.

4. Brooks SA, Martin E, Smeester L, Grace MR, Boggess K and Fry RC: miRNAs as common regulators of the transforming growth factor (TGF) $\beta$ pathway in the preeclamptic placenta and cadmium-treated trophoblasts: Links between the environment, the epigenome and preeclampsia. Food Chem Toxicol 98: 50-57, 2016.

5. Zuckerwise L, Li J, Lu L, Men Y, Geng T, Buhimschi CS, Buhimschi IA, Bukowski R, Guller S, Paidas M, et al: H19 long noncoding RNA alters trophoblast cell migration and invasion by regulating T $\beta R 3$ in placentae with fetal growth restriction. Oncotarget 7: 38398-38407, 2016.

6. Turner RJ, Bloemenkamp KW, Bruijn JA and Baelde HJ: Loss of thrombomodulin in placental dysfunction in preeclampsia. Arterioscler Thromb Vasc Biol 36: 728-735, 2016.

7. van Eerden L, de Groot CJM,Zeeman GG, Page-Christiaens GCM, Pajkrt E, Duvekot JJ, Vandenbussche FP, Oei SG, Scheepers HCJ, van Eyck J,et al: Subsequent pregnancy outcome after mid-trimester termination of pregnancy for preeclampsia. Aust N Z J Obstet Gynaecol: Aug 29, 2017 (Epub ahead of print).

8. Zhu H: Forkhead box transcription factors in embryonic heart development and congenital heart disease. Life Sci 144: 194-201, 2016.

9. Nestal de Moraes G, Bella L, Zona S, Burton MJ and Lam EW: Insights into a critical role of the FOXO3a-FOXM1 Axis in DNA damage response and genotoxic drug resistance. Curr Drug Targets 17: 164-177, 2016.

10. Zona S, Bella L, Burton MJ, Nestal de Moraes G and Lam EW: FOXM1: An emerging master regulator of DNA damage response and genotoxic agent resistance. Biochim Biophys Acta 1839: $1316-1322,2014$

11. Gao F, Bian F, Ma X, Kalinichenko VV and Das SK: Control of regional decidualization in implantation: Role of FoxM1 downstream of Hoxa10 and cyclin D3. Sci Rep 5: 13863, 2015.

12. Xie Y, Cui D, Sui L, Xu Y, Zhang N, Ma Y, Li Y and Kong Y: Induction of forkhead box M1 (FoxM1) by EGF through ERK signaling pathway promotes trophoblast cell invasion. Cell Tissue Res 362: 421-430, 2015.

13. Vaswani K, Hum MW, Chan HW, Ryan J, Wood-Bradley RJ, Nitert MD, Mitchell MD, Armitage JA and Rice GE: The effect of gestational age on angiogenic gene expression in the rat placenta. PLoS One 8: e83762, 2013.

14. Li Y, Zhu H, Klausen C, Peng B and Leung PC: Vascular endothelial growth factor-A (VEGF-A) mediates activin A-induced human trophoblast Endothelial-Like tube formation. Endocrinology 156: 4257-4268, 2015.

15. Francis VA, Abera AB, Matjila M, Millar RP and Katz AA: Kisspeptin regulation of genes involved in cell invasion and angiogenesis in first trimester human trophoblast cells. PLoS One 9: e99680, 2014.

16. Guo X, Feng L, Jia J, Chen R and Yu J: Upregulation of VEGF by small activating RNA and its implications in preeclampsia. Placenta 46: 38-44, 2016.

17. Chomczynski P and Sacchi N: The single-step method of RNA isolation by acid guanidinium thiocyanate-phenol-chloroform extraction: Twenty-something years on. Nat Protoc 1: 581-585, 2006.

18. Gan MF, Yang HL, Qian JL, Wu CS, Yuan CX, Li XF and Zou J: Comparison of two methods for RNA extraction from the nucleus pulposus of intervertebral discs. Genet Mol Res 15, 2016.

19. Livak KJ and Schmittgen TD: Analysis of relative gene expression data using real-time quantitative PCR and the 2(-Delta Delta C(T)) method. Methods 25: 402-408, 2001.

20. Lu Y, Zhang K, Li C, Yao Y, Tao D, Liu Y, Zhang S and Ma Y: Piwil2 suppresses 553 by inducing phosphorylation of signal transducer and activator of transcription 3 in tumor cells. PLoS One 7: e30999, 2012.

21. Shibata Y, Kikuchi R, Ishii H, Suzuki S, Harada K, Hirayama K, Suzuki A, Tatami Y, Kondo K and Murohara T: Balance between angiogenic and anti-angiogenic isoforms of VEGF-A is associated with the complexity and severity of coronary artery disease. Clin Chim Acta 478: 114-119, 2018.

22. Massimiani M, Salvi S, Piccirilli D, Vecchione L, Moresi S, Ferrazzani S, Stuhlmann H and Campagnolo L: A4. EGFL7 in placenta trophoblast and endothelial cells: Implications in the pathogenesis of preeclampsia. J Mater Fetal Neonatal Med 29: 4,2016 .
23. Shah DA and Khalil RA: Bioactive factors in uteroplacental and systemic circulation link placental ischemia to generalized vascular dysfunction in hypertensive pregnancy and preeclampsia. Biochem Pharmacol 95: 211-226, 2015.

24. Nissaisorakarn P, Sharif S and Jim B: Hypertension in pregnancy: Defining blood pressure goals and the value of biomarkers for preeclampsia. Curr Cardiol Rep 18: 131, 2016.

25. Goldman-Wohl DS and Yagel S: Examination of distinct fetal and maternal molecular pathways suggests a mechanism for the development of preeclampsia. J Reprod Immunol 76: 54-60, 2007.

26. Austgulen R: Recent knowledge on mechanisms underlying development of preeclampsia. Tidsskr Nor Laegeforen 124: 21-24, 2004.

27. Xuan RR, Niu TT and Chen HM: Astaxanthin blocks preeclampsia progression by suppressing oxidative stress and inflammation. Mol Med Rep 14: 2697-2704, 2016.

28. Bokslag A, van Weissenbruch M, Mol BW and de Groot CJ: Preeclampsia; short and long-term consequences for mother and neonate. Early Hum Dev 102: 47-50, 2016.

29. Gilani SI, Weissgerber TL, Garovic VD and Jayachandran M: Preeclampsia and extracellular vesicles. Curr Hypertens Rep 18: $68,2016$.

30. Karumanchi SA and Granger JP: Preeclampsia and pregnancy-related hypertensive disorders. Hypertension 67: 238-242, 2016.

31. Liu X, Hu Y, Zheng Y, Liu X, Luo M, Liu W, Zhao Y and Zou L: EPHB4 regulates human trophoblast cell line HTR-8/SVneo function: Implications for the role of EPHB4 in preeclampsia. Biol Reprod 95: 65, 2016

32. Francica P, Nisa L, Aebersold DM, Langer R, Bladt F, Blaukat A, Stroka D, Martínez MR, Zimmer Y and Medová M: Depletion of FOXM1 via MET targeting underlies establishment of a DNA Damage-induced senescence program in gastric cancer. Clin Cancer Res 22: 5322-5336, 2016.

33. Xu MD, Wang Y, Weng W, Wei P, Qi P, Zhang Q, Tan C, Ni SJ, Dong L, Yang Y, et al: A positive feedback loop of IncRNA-PVT1 and FOXM1 facilitates gastric cancer growth and invasion. Clin Cancer Res 23: 2071-2080, 2017.

34. Sayanjali B, Christensen GJM, Al-Zeer MA, Mollenkopf HJ, Meyer TF and Brüggemann H: Propionibacterium acnes inhibits FOXM1 and induces cell cycle alterations in human primary prostate cells. Int J Med Microbiol 306: 517-528, 2016.

35. Maachani UB, Shankavaram U, Kramp T, Tofilon PJ, Camphausen K and Tandle AT: FOXM1 and STAT3 interaction confers radioresistance in glioblastoma cells. Oncotarget 7: 77365-77377, 2016

36. Hackmon R, Pinnaduwage L, Zhang J, Lye SJ, Geraghty DE and Dunk CE: Definitive class I human leukocyte antigen expression in gestational placentation: HLA-F, HLA-E, HLA-C, and HLA-G in extravillous trophoblast invasion on placentation, pregnancy, and parturition. Am J Reprod Immunol: Feb 10, 2017 (Epub ahead of print)

37. Jia Y, Li T, Huang X, Xu X, Zhou X, Jia L, Zhu J, Xie D, Wang K, Zhou Q, et al: Dysregulated DNA Methyltransferase 3A upregulates IGFBP5 to suppress trophoblast cell migration and invasion in preeclampsia. Hypertension 69: 356-366, 2017.

38. Ji L, Brkić J, Liu M, Fu G, Peng C and Wang YL: Placental trophoblast cell differentiation: Physiological regulation and pathological relevance to preeclampsia. Mol Aspects Med 34: 981-1023, 2013.

39. Kin K, Maziarz J, Chavan AR, Kamat M, Vasudevan S, Birt A, Emera D, Lynch VJ, Ott TL, Pavlicev M, et al: The transcriptomic evolution of mammalian pregnancy: Gene expression innovations in endometrial stromal fibroblasts. Genome Biol Evol 8: 2459-2473, 2016.

40. Knöfler M and Pollheimer J: IFPA Award in Placentology lecture: Molecular regulation of human trophoblast invasion. Placenta 33 (Suppl): S55-S62, 2012.

41. Roberts E, Cossigny DA and Quan GM: The role of vascular endothelial growth factor in metastatic prostate cancer to the skeleton. Prostate Cancer 2013: 418340, 2013.

42. Ahmed A, Rezai H and Broadway-Stringer S: Evidence-Based revised view of the pathophysiology of preeclampsia. Adv Exp Med Biol 956: 355-374, 2017.

This work is licensed under a Creative Commons Attribution-NonCommercial-NoDerivatives 4.0 International (CC BY-NC-ND 4.0) License. 